\title{
Effects of Brief Mindfulness Meditation on Attention Switching
}

\author{
Tomasz Jankowski ${ }^{1}$ (D) Pawel Holas ${ }^{2}$
}

Published online: 10 February 2020

(C) The Author(s) 2020

\begin{abstract}
Objectives Attention switching is postulated in many theories as a crucial executive function related to mindfulness. However, the existing literature does not provide convincing support for this claim. Based on the attentional control theory, the presented experiment tested the hypothesis that brief mindfulness training improves attention switching in the presence of anxiety.

Methods Seventy-four undergraduates $(53$ women) with an average age of 22.25 years $(\mathrm{SD}=2.42)$ performed a pre-test switching task, watched an emotional film clip, and then were randomly assigned to three conditions: brief mindfulness training, worry induction, and free mind-wandering, followed by a post-test switching task.

Results We found reduced overall reaction times (i.e., for both switch and no-switch trials) in mindfulness compared with worry and free mind-wandering conditions $\left(F[2,68.9]=5.09, p=.009, \eta^{2}=.13\right)$, but no differences between conditions with regard to the switch cost.

Conclusions The results are discussed in the context of working memory capacity and processing speed theory. We suggest that mindfulness may release attentional resources otherwise engaged in the inhibition of task-irrelevant thinking associated with anxiety and thus facilities the general efficiency of cognitive processes. Suggestions for future research are proposed.
\end{abstract}

Keywords Mindfulness $\cdot$ Attention switching $\cdot$ Worry $\cdot$ Anxiety $\cdot$ Processing speed

Mindfulness has been one of the most investigated phenomena for the last decade. The most common understanding of this concept defines it as the state of receptive, open, and nonjudgmental attention focused on the present (Kabat-Zinn 2003). Direct reference to attentional processes expressed in the definition of mindfulness has prompted scientists to publish theoretical speculations and empirical studies examining its relation with attentional control. Among various processes included in the broad concept of attentional control, attention switching is considered being one of the most important to maintain the mindfulness state (Bishop et al. 2004; Holas and Jankowski 2013).

Attention switching is one of executive functions and concerns "shifting back and forth between multiple tasks, operations, or mental sets" (Miyake et al. 2000, p. 55). It is a complex cognitive ability and includes at least two complementary

Tomasz Jankowski tojankowski@kul.pl

1 Institute of Psychology, The John Paul II Catholic University of Lublin, Al. Racławickie 14, 20-950 Lublin, Poland

2 University of Warsaw, Warsaw, Poland processes: inhibition of previously activated task-set features interfering with the current task, and reconfiguration or retrieving from the long-term memory a new task set (Vandierendonck et al. 2010).

The performance efficiency in switching tasks is measured with reaction times (RTs) that - depending on a procedurereveal several effects. One of the most important effects is related to the switch cost and is operationalized as a difference between an average RT in switch-between-tasks trials and an average RT in no-switch trials (Monsell 2003). Thus, the smaller switch cost, the better efficiency of the executive function. The second effect is observed in procedures including blocks with "pure" trials (only one task in a single block) and blocks with "mixed" trials (two or more task in a single block). This effect was called "no-switch cost" because there is observed a difference in averaged RTs between "pure" and "mixed" blocks even in no-switch responses. It is interpreted as revealing uncertainty associated with expecting or preparing for a switch (Salthouse et al. 1998) or necessity "to maintain two task-sets in an available state rather than one" (Rogers and Monsell 1995, p. 216). In other words, the no-switch cost is associated rather with acting in a dual-task condition compared with a single-task condition than with attention switching. Lastly, RTs in a switching task can be affected by 
processing speed (Salthouse et al. 1998), i.e. a general, nonspecific ability that affect performance in a lot of cognitive tasks. Processing speed is related to the rate with which many elementary cognitive operations can be executed (Salthouse 1996). In conclusion, RTs in a switching task depend on the switch cost (which reveals efficiency of attention switching), the no-switch cost (which reveals performance efficiency for a dual-task condition), and processing speed (which reveals the generic ease to perform cognitive tasks).

Relationship between switching attention and mindfulness seems to be reciprocal. On the one hand, efficient attention switching allows for cognitive flexibility which seems important for a mindful observation of ongoing and changing experiences (Bishop et al. 2004; Holas and Jankowski 2013). Every-day, spontaneous mindfulness state, understood as receptive and open awareness and attention, requires the ability to switch between global and local perception (Brown et al. 2007). During mindfulness meditation, switching attention is needed even more, for example, when a person, particularly in the beginning of a practice, becomes aware of mindwandering and switch attention back to breath or another object (Holas and Jankowski 2013). Thus, efficient switching attention facilitates induction and maintenance of the mindfulness state. On the other hand, one could ask whether mindfulness facilitates switching attention. Some theoretical models postulate such effects. For example, according to the metacognitive model of mindfulness (Jankowski and Holas 2014), a regular practice improves the overall level of executive functions, including switching attention, just as the practice of self-control improves the overall level of selfregulation (e.g., Hui et al. 2009).

The reviews by Chiesa et al. (2011) and Chiesa and Serretti (2010) showed, however, that relations between mindfulness and executive attention are not as obvious as theoretical models of mindfulness have claimed. While some studies suggested significant improvement in executive attention (e.g., Jha et al. 2007; Wenk-Sormaz 2005), others revealed no impact of mindfulness training (MT) (e.g., Anderson et al. 2007; Polak 2009). This ambiguity among findings is noticeable regarding to the effect of MT on the switch cost. While there is a strong theoretical justification for a hypothesis which says the practice of mindfulness improves this aspect of executive attention, there is also very weak empirical proof for it. We are aware of five published studies that examined this hypothesis and their results are ambiguous. For example, Anderson et al. (2007) compared participants randomly assigned to an eight-week mindfulnessbased stress reduction (MBSR) program or to a waiting list and found no significant effect of MBSR on attention switching. Similarly, Lykins et al. (2012) compared a group of experienced, long-term meditators with a group of non-meditators and also reported no significant differences between those groups in the efficacy of attentional switching. In two other studies, although no reductions in the switch cost were found, some improvements suggesting the positive impact of mindfulness on cognitive flexibility were reported. Chambers et al. (2008) revealed for example, that after a 10-day intensive mindfulness meditation retreat, participants' overall RTs in a switching task decreased, which was not the case for a wait-list group. In the second study, Heeren, Broeck, and Philippot (2009) found no differences between participants who completed mindfulnessbased cognitive therapy (MBCT) and matched control individuals in the task associated with the set-switching cost. The MBCT group showed, however, improvement in the verbal fluency task which suggests that MT influenced participants' cognitive flexibility (Heeren et al. 2009).

The only study, to our knowledge, that evidenced a positive relationship between mindfulness and attention switching was carried out by Hodgins and Adair (2010) who compared the ability to shift perspectives in adult meditators versus nonmeditators. In this task, individuals were presented with three drawings. Each of the drawings could be seen in two different ways, for example, it could be interpreted as a duck versus a rabbit - dependent on the perspective a participant took. Participants were instructed to identify the first image as fast as possible; then they watched the drawing again and this time they had to change a perspective to identify the second image. Results showed that meditators identified the first image faster than non-meditators. There were no differences between groups in reaction times for the second viewing of the image; however, meditators identified a greater number of alternative perspectives compared with non-meditators.

Taking the ambiguity of the findings presented above into account, we posit that a brief mindfulness meditation (BMM) could improve attention switching particularly in those persons whose switching abilities have been previously diminished by stress or other negative emotions. This hypothesis is based on the attentional control theory (Eysenck et al. 2007) which states that anxiety (particularly its cognitive aspect, i.e. worrying) consumes cognitive resources and decreases efficiency (measured by RTs) and/or accuracy during a task demanding effort. Because BMM leads to a reduction in anxiety and worry (e.g. Call et al. 2014), we postulate that BMM may improve attentional switching indirectly, through relieving working memory from maladaptive thinking. Therefore, the present study tested the effect of BMM on switching attention in the stress condition. We used the design applied in similar investigations (e.g., Polak 2009; Wenk-Sormaz 2005), but modified it to focus on our hypothesis.

\section{Method}

\section{Participants}

Eighty-one participants (53 women) with an average age of 22.25 years $(\mathrm{SD}=2.42)$ were recruited through 
advertisements at local universities. They volunteered to participate in the study in return for a cinema voucher. Informed consent was obtained from all individual participants included in the study. All had normal vision and no difficulty differentiating colors. Participants were randomly assigned to three experimental conditions. Four participants were excluded from the analysis due to a technical difficulty recording their data. Three participants were also excluded because they showed poor understanding of experimental instructions and made a significant number of mistakes $(32-48 \%)$. The final number of participants was 74 (28 in the mindfulness group, 21 in the worry group, and 25 in the control-free mind-wandering group).

\section{Procedures}

\section{Negative Affect Induction}

To induce anxiety, we used a scene from the well-known film Misery, in which Annie - one of the main characters - breaks Paul's (another main character) legs. The scene was chosen because in laboratory settings it proved to evoke negative affect including high level of arousal and fear as well as a little bit of disgust (Schaefer et al. 2010).

\section{Mindfulness State Manipulation}

We developed brief mindfulness meditation based on classic mindfulness instructions used in the MBSR (Kabat-Zinn 1990). It was recorded by experienced mindfulness teacher. The recording led participants through a 10-minute mindfulness exercise during which it instructed them to observe every aspect of current experience such as thoughts and feelings with acceptance and curiosity. If these mental events emerge, participants were further instructed to let them go and gently redirect their attention to breathing sensation in the present moment. The guided meditation included instructions such as "allowing any thoughts or feelings that occur to rise and fall, without trying to hold on to them or get rid of them... just continue bringing your awareness to your experience in this moment."

\section{Worrying State Manipulation}

The worrying group completed a task which was intended to evoke worrying explicitly. We adapted a procedure used in Butler, Wells, and Dewick's study (1995). Participants assigned to the worry condition did a 10-min exercise introduced to them as a mental preparation for the next part of the experiment. Similar to the mindfulness group, they listened to the same male voice instructing them on what to do. Participants were asked to mentally assess their emotions related to the film scene they had already seen as pleasant or unpleasant, to think about how they could be evaluated by others if they expressed fear while watching a film, and to consider how they might feel if they watched this as a real scene instead of a fictional portrayal.

\section{Free Mind-Wandering Condition}

Subjects in the free mind-wandering condition were instructed to sit and wait for the next part of the experiment with their mind wandering freely, as it usually does when waiting for something. After $10 \mathrm{~min}$, they were asked to perform the next task.

\section{Switching Task}

A task-cueing paradigm was applied to measure the switch cost (Monsell 2003). Each participant performed the task on a personal computer (Dell) with a 15 -in. monitor $(60-\mathrm{Hz}$ vertical refresh rate). Participants were seated about $50 \mathrm{~cm}$ from the screen mounted immediately in front of them. The task was completed using E-Prime 2.0 software. The target stimuli consisted of 120, 1-2 syllable words; 20 of these referred to names of plants and 100 were various nouns not related to plants. The stimuli were displayed at the center of the screen in four different colors (blue, red, green, and teal) in Courier New font (bold, font size 32) on a white background.

The task consisted of four blocks - three were used to practice and the last one was a test block. The first block included 6 trials and participants at each time decided whether the word was a plant or not. As for the second block, participants decided in each of the 6 trials whether the word was displayed in red or a different color. The third block consisted of 8 trials. For one half of the trials participants were to make lexical decisions ("plant or not"), while for the other half of the trials, they were to decide on the color of a displayed word ("red or not"). The last block consisted of 100 trials and participants received the same instruction as they had in the third block. Words were presented randomly for each participant in every block and they appeared only once during the whole switching task. Participants responded using both hands, with index and ring fingers, using four different keys of a standard QWERTY keyboard as follows: " $w$ " if the word referred to a plant and "s" if not, "p" if the word was displayed in red and "l" if in another color (pairs of keys - on the left or right side of the keyboard-referred to a particular task and were counterbalanced among participants). For both the practice and test blocks, specific task reminders were displayed on top of the screen: "Plant?" on the left side and "Red?" on the right side (sides were counterbalanced among participants and were on the same side as the relevant response keys). In the single trial, the task was cued using a rectangle-framed reminder with the word "Plant" for a lexical decision or "Red" for a color decision. At the beginning of the switch task and 
before each of the blocks, participants were instructed about target stimuli and about the task itself.

The task procedure is presented in Fig. 1. A single trial started with a cue that was displayed on top of the screen and remained there until the end of a trial. One hundred and fifty milliseconds after cueing, a stimulus appeared at the center of the screen and was visible until the given participant responded. Then, if a participant gave a wrong answer, the feedback "WRONG" was presented on the bottom of the screen for $1850 \mathrm{~ms}$; if a participant gave a correct answer, a blank screen was displayed for the same amount of time. There were two probe types in a test block: no-switch (when the trial was preceded by a trial with the same task) and switch (when the trial was preceded by a trial with a different task).

Each participant took part in the investigation individually. After being greeted in the laboratory, she or he was seated in a private cubicle. The experiment was introduced to participants as a "study on cognitive and emotional processes." An experimenter additionally explained to them that an important part of the study was going to be a fictional scene presenting a lot of pain and that they were allowed to resign from the experiment at any time they wished. After giving their consent, the subjects were instructed to do all blocks of the switch task and the experimenter left the room. The results from this pre-test were further treated as a baseline level of the performance for each of the participants. When finished, they were asked to put headphones on, whereupon the film inducing a negative mood started. After about $3 \mathrm{~min}$, the film ended and participants, based on prior random assignment to three different conditions, listened to mindfulness vs. worry vs. free mindwandering instructions. This part of the experiment lasted about $10 \mathrm{~min}$. Immediately after it finished, an experimenter entered the room and asked participants to complete the test block of the switch task the second time. Finally, they were debriefed and awarded a cinema ticket. The whole session took about $50 \mathrm{~min}$.

\section{Manipulation Check}

We conducted two pilot studies prior to the main experiment to check whether inductions of mood and mindfulness were effective, because we did not want to interrupt the procedure to perform manipulation control. In the first pilot study, 18 participants completed PANAS (Watson and Clark 1999) to measure negative affect, and they were then randomly assigned to two conditions: in the first, they watched the whole scene and, in the second, they watched the same scene but with the exclusion of the last few seconds which presented an act of violence. This last scene was crucial for inducing negative affect. Finally, participants completed PANAS once again. In spite of randomization, the groups differed in pre-test at the level of negative affect $(p=.09)$ and fear $(p=.03)$; therefore, we used a two-way mixed ANOVA with one betweengroup variable (mood manipulation: negative affect vs. neutral) and one within-subject variable (measurement time: pre-test vs. post-test). There was significant interaction effect for fear, $F(1,16)=5.36, p=.034, \eta^{2}=.25$, and marginal interaction effect for negative affect, $F(1,16)=$ $3.43, p=.09, \eta^{2}=.18$. Simple effects indicate that an increase in fear $(p=.002)$ and in negative affect $(p=.01)$ from pre-test to post-test was reported only in the group watching the whole scene of violence. The results suggest that the film evokes mainly emotion of fear.

To check BMM effect, we conducted the second pilot study $(N=48)$. Participants were randomly assigned to the mindfulness, worry, or free mind-wandering groups. Because we wanted to test the efficacy of mindfulness and worry inductions directly after exposition to an aversive stimulus, participants were briefly introduced to the study and then watched the emotional film clip. After induction, they immediately listened to the instructions about either mindfulness or worrying. Next, they were asked to complete the Toronto Mindfulness Scale including a decentration scale (Lau et al. 2006) and PANAS (Watson and Clark 1999). Each of the
Fig. 1 The switching task procedure

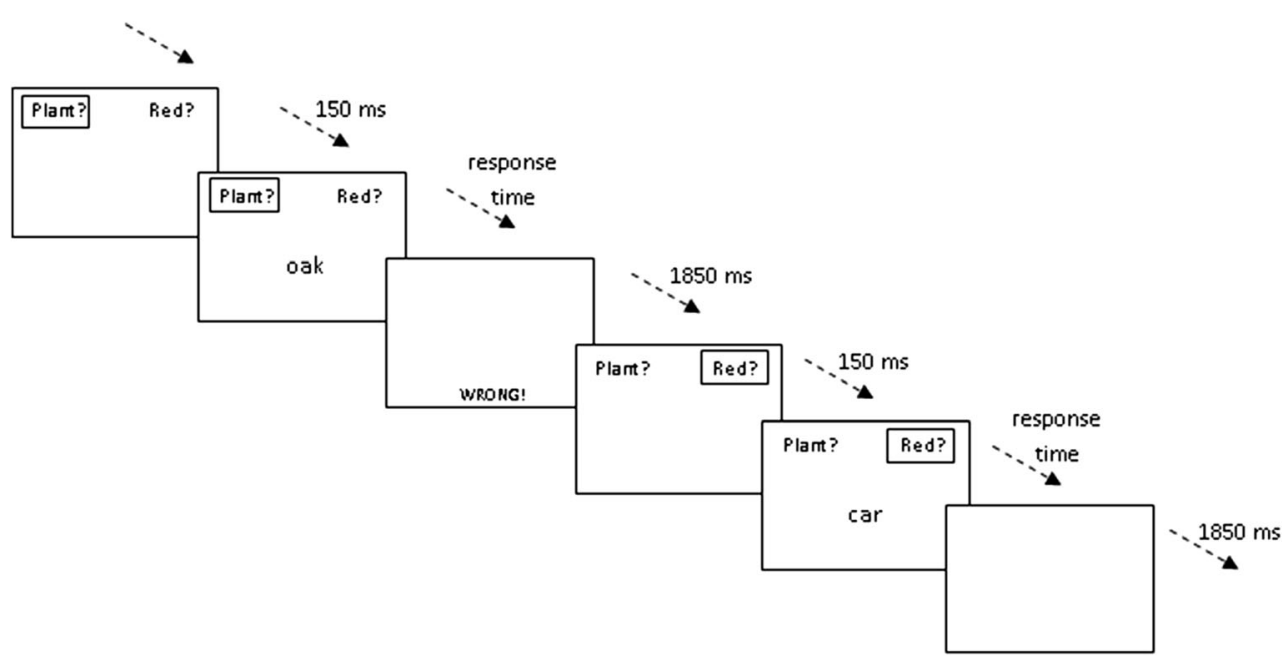


dependent variables was analyzed using a one-way ANOVA. An overall effect of group was observed for the mindfulness state, $F(2,45)=5.5, p=.007, \eta^{2}=.20$; no effect of group was observed for positive and negative affect. Furthermore, the planned contrasts revealed that the mindfulness group was more decentered during the task compared with both control groups, $t(45)=3.3, p=.002$. This result suggests that BMM does not change emotions, but impacts the way participants approach their experiencefrom being immersed in the experience in control groups to being decentered in a mindfulness group.

\section{Data Analyses}

We analyzed RTs in an attention switching task with mixed effects modeling (Baayen et al. 2008; Baayen and Milin 2010). This approach is recommended for several reasons. Above all, it helps with the problem of "language-as-fixedeffect-fallacy" which comes from aggregating data for each subject over the same types of items (e.g., "switch" vs "noswitch") and using these means in ANOVA (Clark 1973; Raaijmakers et al. 1999). The traditional approach based on ANOVA overestimates standard errors which results in a higher risk of type I error. Mixed effects modeling solves this problem and provides several additional benefits. In tasks with a dozen probes, several important effects might influence the variability of RTs, such as effects of learning, fatigue, or the preceding trial (Baayen et al. 2008). Explicitly taking these confounding effects into account by entering them into a multilevel regression allows for an increase in the test power to detect subtle target effects.

Therefore, we analyzed a hierarchical mixed effects regression model including several fixed and random effects. First, we entered covariates from the first level of data (i.e., withinsubject level) such as a baseline switch cost effect (i.e., averaged RTs for each participant and a probe type at pre-test), a preceding trial RT, and a linear and non-linear (squared) trial number as fixed effects. We also allowed the intercept in a model to vary across subjects and words (crossed random effects). Second, we entered a predictor from the second level of data, i.e. between-subject condition (a group: mindfulness vs. free mind-wandering vs. worry) and a predictor from the first level of data, i.e. within-subject condition (a probe type: switch vs. no-switch) as additional fixed effects. We also allowed the effect of a probe type to vary across subjects (additional random effect). Finally, we also included an interaction effect between a group and a probe type. Before analysis, we removed incorrect responses (about 3\%), RTs exceeding the range of 300-3000 $\mathrm{ms}$ (about $0.5 \%$ ), and logtransformed RTs. We did not group mean center covariates because we wanted to partial out their effects on both level 1 and level 2, keeping the model relatively simple (Enders and Tofighi 2007). All analyses were performed using $\mathrm{R}$ ( $\mathrm{R}$ Core Team 2014) with lme4 (Bates et al. 2015), sjPlot (Lüdecke 2018), and emmeans (Lenth et al. 2018) packages.

\section{Results}

Table 1 presents comparison of the models fit at each of the three steps of multilevel hierarchical regression analysis. Fixed effects related to the covariates at the first step explained $28 \%$ of RT variability in the attention switching task at the post-test. Including a probe type and a group as predictors at the second step was associated with significant model fit improvement $\left(\Delta \chi^{2}(5)=38, p<.001, \Delta A I C=-28, \Delta R^{2}=\right.$ $0.021)$, but entering the interaction term between a group and a probe type at the third step did not significantly improve model fit $\left(\Delta \chi^{2}(2)=1.47, p=.48, \Delta A I C=2.6, \Delta R^{2}=0.001\right)$.

In Tables 2 and 3, we present results for the second step of the model analysis and estimated marginal means for RTs by probe types and groups, respectively. We observed significant effects of the baseline switch cost $(F[1734.5]=809.62$, $\left.p<.001, \eta^{2}=.52\right)$, preceding trial RT $(F[16,715.8]=69.46$, $\left.p<.001, \eta^{2}=.01\right)$, and group $(F[2,68.9]=5.09, p=.009$, $\left.\eta^{2}=.13\right)$. Neither trial number nor probe type was significant predictors of the RTs in the post-test switching attention task $\left(F[2,6828.2]=.90, p=.406, \eta^{2}<.001\right.$ and $F[1,72.6]=1.18$, $p=.28, \eta^{2}=.02$, respectively). We suspected that the baseline (pre-test) switch cost effect was responsible for the disappearance of probe type and trial number effects at the post-test. Therefore, we repeated the analysis without baseline RTs as an independent variable to check whether the remaining effects approached the alpha level of .05. This time, results showed significant probe type effect $(F[1,6868.2]=$ $\left.34.84, p<.001, \eta^{2}=.005\right)$ but still no significant effects of the trial number, i.e., practice $(F[2,6860.1]=1.37$, $\left.p=.253, \eta^{2}<.001\right)$. Because the effect of trial number was observed in the pre-test task $(F[2,7286.3]=56.27$, $p<.001, \eta^{2}=.02$ ) and was non-linear (RTs approached asymptotically to the low, stable level as the number of trials increased), we suggest that in the post-test, the practice effect was not observed because of the floor effect.

Figure 2 displays estimated marginal RT means with the standard errors for mindfulness, free mind-wandering, and worry groups. Analysis of simple effects (with Tukey adjustment for comparing three estimates) showed that the BMM

Table 1 Comparison of models in hierarchical regression analysis

\begin{tabular}{llllrrr}
\hline Model & Df & AIC & \multicolumn{1}{l}{ BIC } & $\Delta \chi^{2}$ & $\Delta D f$ & \multicolumn{1}{c}{$p$} \\
\hline 1 & 8 & $98,952.87$ & $99,007.68$ & - & - & - \\
2 & 13 & $98,942.09$ & $99,031.16$ & 20.78 & 5 & .001 \\
3 & 15 & $98,945.25$ & $99,048.02$ & 0.84 & 2 & .66 \\
\hline
\end{tabular}


Table 2 Results for the second step of the hierarchical regression analysis (log-transformed RTs)

\begin{tabular}{llll}
\hline Fixed effects & $F$ & $D f$ & $p$ \\
Trial number & 1.74 & 2 & .38 \\
Preceding probe & 128.78 & 1 & .001 \\
Baseline RT & 563.56 & 1 & .001 \\
Probe type & 0.55 & 1 & .42 \\
Group & 4.43 & 2 & .01 \\
Random effects & & & \\
$\sigma^{2}$ & 0.09 & & \\
$\tau_{00}$ Word & 0.001 & & \\
$\tau_{00}$ Subject & 0.01 & & \\
$\tau_{11 \text { Subject: Probe Type }}$ & 0.001 & & \\
$\rho_{01 \text { Subject }}$ & -0.31 & & \\
ICC Word & 0.02 & & \\
ICC Subject & 0.08 & & \\
Observations & 6984 & & \\
Marginal $^{2} /$ conditional $R^{2}$ & $.304 / .379$ & & \\
\hline
\end{tabular}

reduced RTs compared with worry $(\triangle R T=-65 \mathrm{~ms}, t=-$ $3.02, p=.008)$ and mind-wandering instructions $(\Delta R T=-$ $41 \mathrm{~ms}, t=-2.32, p=.053)$. The difference between the mind-wandering and worry effects was not significant $(\Delta R T=-24 \mathrm{~ms}, t=-.81, p=.99)$.

\section{Discussion}

The aim of the present study was to investigate whether BMM improves attention switching under stressful conditions. Based on attentional control theory (Eysenck et al. 2007), we assumed that anxiety and worrying deplete attentional resources and reduce efficiency in cognitive tasks demanding effort, such as a switching task. Therefore, we expected that, following negative mood induction, BMM would reduce the switch cost by relieving working memory of its temporary load caused by irrelevant mental processes such as worry.

Table 3 Estimated marginal means for reaction times (in $\mathrm{ms}$ ) by probe types and groups

\begin{tabular}{llllll}
\hline Probe type & Group & Mean & SE & \multicolumn{2}{l}{$95 \%$ confidence interval } \\
\cline { 3 - 6 } & & & & Lower & Upper \\
\hline \multirow{2}{*}{ No-switch } & Mindfulness & 779 & 16 & 748 & 811 \\
& Rest & 821 & 16 & 788 & 853 \\
& Worry & 845 & 18 & 810 & 880 \\
Switch & Mindfulness & 772 & 17 & 739 & 805 \\
& Rest & 813 & 17 & 780 & 846 \\
& Worry & 837 & 18 & 802 & 873 \\
\hline
\end{tabular}

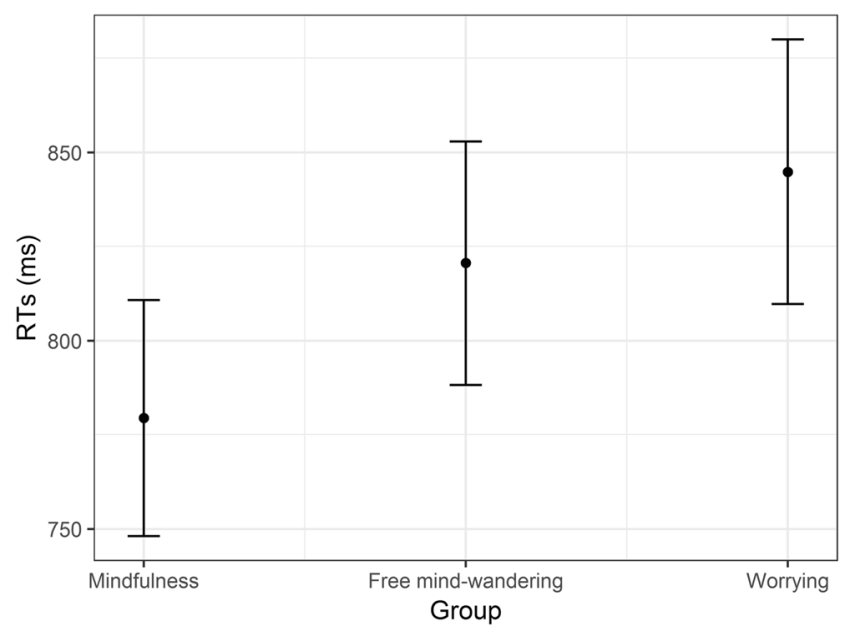

Fig. 2 Estimated marginal means for the general speed of processing (RTs) in the switching task by experimental groups

The results of the current study did not support our hypothesis. We did not observe a significant influence of BMM on the size of the switch cost. A difference in RTs between switch and no-switch trials was the same regardless the experimental manipulation. This suggests that mindfulness evoked in a stressful condition did not improve attention switching in our study. However, we observed reduced overall RTs in a switching attention task under BMM condition. This means that although the switch cost was the same in all groups, RTs for both switch and no-switch trials were faster in the mindfulness group compared with the worry and mind-wandering groups.

The results found by us are consistent with findings of the Chambers et al. (2008) study, who observed that people after 10 days of mindfulness training had faster RTs in the switching attention task, compared with those of the wait-list group. Our study suggests that the effect observed by Chambers et al. (2008) is significant even after a brief, 10-min mindfulness exercise, if preceded by induction of a negative affect. The question is what other cognitive processes - if not attention switching - are influenced by BMM during the switching task?

As we mentioned in the introduction, at least three effects influencing RTs in the switching attention task can be identified: the switch cost, the no-switch cost, and an overall processing speed. Since we did not observe a significant reduction of the switch cost in the mindfulness group, one or both of the other effects may be responsible for the faster RTs related to BMM. Unfortunately, the version of the switching task we used does not allow us to resolve this issue in the current study. To distinguish the no-switch cost from processing speed effect, future research should use a procedure including "pure" and "mixed" trial blocks. If BMM affected overall RTs in both one-task and in dual-task conditions, an explanation referring to speed processing would be more likely. However, if BMM affected overall RTs in a dual-task condition but not in a one- 
task condition, then an explanation referring to the no-switch cost would be more plausible.

Regardless of the interpretations proposed above, a further important question arises: why overall RTs in the switching task are faster after $10 \mathrm{~min}$ of brief mindfulness training carried out after induction of negative affect? We assumed that (a) negative affect, and anxiety in particular, leads to worrying (Eysenck et al. 2007); (b) worrying, like mind-wandering, overloads working memory capacity (WMC) (Trezise and Reeve 2016) and the phonological loop in particular (Beilock et al. 2007); and (c) WMC affects attention switching (Vandierendonck et al. 2010). Since several studies found that mindfulness is negatively related to worrying (e.g., Fisak and Lehe 2011; for review see $\mathrm{Gu}$ et al. 2015), we suggest the following explanation: BMM may release resources related to WMC and thus it may improve efficiency of the switching task, compared with the mind-wandering and worrying groups. Although we did not measure WMC, such an interpretation is supported by the results of other researchers. For example, Jha et al. (2010) found that regular mindfulness practice during an intensive 1-month retreat protects against depletion of WMC in soldiers under prolonged high-stress conditions compared with controls. The authors interpreted these results in line with our proposition stating that mindfulness mitigates detrimental effects of stress on WMC and enhances cognitive processing (Jha et al. 2010).

Explanations of faster RTs in BMM group referring to the no-switch cost or processing speed agree with the proposed impact of mindfulness on WMC. The no-switch cost is related to WMC by definition, because efficient performance in a switch-between-tasks condition requires maintaining both task-sets in WMC. Thus, releasing cognitive resources by BMM may make task-sets more available and facilitates performance. Contrary, overload of WMC caused by worrying may make both task-sets less available and increases the noswitch cost. Results of Baddeley's et al. (2001) study support such a possibility. They found that articulatory suppression (a component of the phonological loop) increased differences in RTs between mixed and pure blocks compared with the control group without suppression. In other words, the group with suppressed phonological loop presented the higher no-switch cost. Similarly, Beaudreau and O'Hara (2009) found that anxiety diminishes processing speed and Conway et al. (2002) suggest that processing speed relates to WMC.

We suggest that brief mindfulness exercises may promote taking a decentered attitude toward an experience under stressful conditions and in that way, they may diminish cognitive interference of intrusive processes. Our proposition is based on results from the main experiment and pilot studies conducted prior to it. First, we assume that our manipulations (mood induction, worrying induction) were effective. We used a modified version of Butler et al.'s (1995) instruction for worry induction, which in their study evoked worrying thoughts up to 3 days after the experimental manipulation. Results of our pilot study showed that the film we used in the main experiment as a mood induction evoked negative affect and anxiety in particular. Therefore, we expected an increased probability of spontaneous worrying as a cognitive aspect of anxiety in the free mind-wandering condition. In the second pilot study, we found that mindfulness meditation increased decentered attitude toward an experience. Therefore, the hypothesis one could test in the future research directly is that decentration mediates the influence of BMM on processing speed in the switching task.

\section{Limitations and Future Research}

Our study has several limitations. We did not include a condition with neutral emotions - that is, without evoking negative emotions. It would be helpful to test if participants after BMM would still be overall faster than subjects in a free mindwandering condition in a neutral emotional state. However, we think this is not crucial for verification of our hypothesis, because we assumed that the positive effect of a brief mindfulness exercise on cognitive processing can be observed mainly in a stressful context. If we are correct, then the majority of previous research has failed to observe an increase in efficiency of cognitive processing due to BMM, because these studies did not include induction of negative emotions before a target task. Studies using a more complex design, including both emotional and non-emotional conditions, are needed to gain more insight into the effects and mechanisms of mindfulness meditation on switching attention. We did not control for emotions and mindfulness level after each stage of the main study; we did this purposefully to prevent the interruption of subtle effects of manipulations. Instead, we controlled those variables in preceding pilot studies. However, future studies would benefit from using non-intrusive measures (e.g., psychophysiological) to check mediational effects related to experimental manipulations.

The current results suggest that individuals in a stressful condition following BMM might not reduce the switch cost in post-test; nevertheless, their reaction times, regardless of probe type (switch vs. no-switch), were overall faster than in control conditions. We believe that this result indicates a specific beneficial effect of mindfulness practice, even brief, on cognitive functioning. Mindfulness - understood as a temporary state - might not improve executive functions, at least in novice meditators. Instead, being mindful during a cognitive task may allow for the better use of attentional resources in the face of negative emotions. Although our findings are not definitely clear, in line with the claim by Prakash et al. that contradicting findings relating to mindfulness effects on attention need further rigorous explorations (Prakash et al. 2018), we 
believe that our study points at one of important directions for future research in this domain.

Acknowledgments We thank Magdalena Bąk and Paulina Staszczak for their help with conducting the study.

Author Contributions TJ: designed and executed the study, analyzed the data, and wrote the paper. PH: collaborated in the designing, writing, and editing of the final manuscript. Both authors approved the final version of the manuscript for submission.

Funding Information This research was financially supported by grant from the National Science Centre, Poland: N N106 135137 to Tomasz Jankowski and in part by a BST grant no $1868 / 16$ of the University of Warsaw to Pawel Holas.

Data Availability Data are available as supplemental materials attached to the published paper.

\section{Compliance with Ethical Standards}

Conflict of Interest The authors declare that they have no conflict of interest.

Ethics Statement All procedures followed were approved by the Ethical Review Board (ERB) of the Institute of Psychology in the John Paul II Catholic University of Lublin as being in accordance with the ethical standards of the ERB and with the Helsinki Declaration of 1964 and its later amendments.

Informed Consent Statement Informed consent was obtained from all participants for being included in the study.

Open Access This article is licensed under a Creative Commons Attribution 4.0 International License, which permits use, sharing, adaptation, distribution and reproduction in any medium or format, as long as you give appropriate credit to the original author(s) and the source, provide a link to the Creative Commons licence, and indicate if changes were made. The images or other third party material in this article are included in the article's Creative Commons licence, unless indicated otherwise in a credit line to the material. If material is not included in the article's Creative Commons licence and your intended use is not permitted by statutory regulation or exceeds the permitted use, you will need to obtain permission directly from the copyright holder. To view a copy of this licence, visit http://creativecommons.org/licenses/by/4.0/.

\section{References}

Anderson, N. D., Lau, M. A., Segal, Z. V., \& Bishop, S. R. (2007). Mindfulness-based stress reduction and attentional control. Clinical Psychology, 463, 449-463. https://doi.org/10.1002/ cpp.544.

Baddeley, A. D., Chincotta, D. M., \& Adlam, A. (2001). Working memory and the control of action: evidence from task switching. Journal of Experimental Psychology: General, 130, 641-657. https://doi. org/10.1037/0096-3445.130.4.641.

Bates, D., Maechler, M., Bolker, B., \& Walker, S. (2015). Fitting linear mixed-effects models using lme4. Journal of Statistical Software, 67(1), 1-48. https://doi.org/10.18637/jss.v067.i01.
Baayen, R. H., Davidson, D. J., \& Bates, D. M. (2008). Mixed-effects modeling with crossed random effects for subjects and items. Journal of Memory and Language, 59, 390-412. https://doi.org/ 10.1016/j.jml.2007.12.005.

Baayen, R. H., \& Milin, P. (2010). Analyzing reaction times. International Journal of Psychological Research, 3, 12-28. https:// doi.org/10.21500/20112084.807.

Beaudreau, S. A., \& O'Hara, R. (2009). The association of anxiety and depressive symptoms with cognitive performance in communitydwelling older adults. Psychology and Aging, 24, 507-512. https:// doi.org/10.1037/a0016035.

Beilock, S., Rydell, R. J., \& McConnell, A. R. (2007). Stereotype threat and working memory: mechanisms, alleviation, and spillover. Journal of Experimental Psychology, 136, 256-276. https://doi. org/10.1037/0096-3445.136.2.256.

Bishop, S. R., Lau, M., Shapiro, S., Carlson, L., Anderson, N., Carmody, J., Segal, Z., Abbey, S., Speca, M., Velting, D., \& Devins, G. (2004). Mindfulness: a proposed operational definition. Clinical Psychology: Science and Practice, 11, 230-241. https://doi.org/10. 1093/clipsy.bph077.

Brown, K. W., Ryan, R. M., \& Creswell, J. D. (2007). Mindfulness: theoretical foundations and evidence for its salutary effects. Psychological Inquiry, 18, 211-237. https://doi.org/10.1093/ clipsy.bph077.

Butler, G., Wells, A., \& Dewick, H. (1995). Differential effects of worry and imagery after exposure to a stressful stimulus: a pilot study. Behavioural and Cognitive Psychotherapy, 23, 45-56. https://doi. org/10.1017/S1352465800017628.

Call, D., Miron, L., \& Orcutt, H. (2014). Effectiveness of brief mindfulness techniques in reducing symptoms of anxiety and stress. Mindfulness, 5, 658-668. https://doi.org/10.1007/s12671-0130218-6.

Chambers, R., Lo, B. C. Y., \& Allen, N. B. (2008). The impact of intensive mindfulness training on attentional control, cognitive style, and affect. Cognitive Therapy and Research, 32, 303-322. https://doi. org/10.1007/s10608-007-9119-0.

Chiesa, A., Calati, R., \& Serretti, A. (2011). Does mindfulness training improve cognitive abilities? A systematic review of neuropsychological findings. Clinical Psychology Review, 31, 449-464. https:// doi.org/10.1016/j.cpr.2010.11.003.

Chiesa, A., \& Serretti, A. (2010). A systematic review of neurobiological and clinical features of mindfulness meditations. Psychological Medicine, 40, 1239-1252. https://doi.org/10.1017/ S0033291709991747.

Clark, H. H. (1973). The language-as-fixed-effect fallacy: a critique of language statistics in psychological research. Journal of Verbal Learning \& Verbal Behavior, 12, 335-359. https://doi.org/10.1016/ S0022-5371(73)80014-3.

Conway, A. R. A., Cowan, N., Bunting, M. F., Therriault, D. J., \& Minkoff, S. R. B. (2002). A latent variable analysis of working memory capacity, short-term memory capacity, processing speed, and general fluid intelligence. Intelligence, 30, 163-184. https:// doi.org/10.1016/S0160-2896(01)00096-4.

Enders, C. K., \& Tofighi, D. (2007). Centering predictor variables in cross-sectional multilevel models: a new look at an old issue. Psychological Methods, 12, 121-138. https://doi.org/10.1037/ 1082-989X.12.2.121.

Eysenck, M. W., Derakshan, N., Santos, R., \& Calvo, M. G. (2007). Anxiety and cognitive performance: attentional control theory. Emotion, 7, 336-353. https://doi.org/10.1037/1528-3542.7.2.336.

Fisak, B., \& Lehe, A. C. (2011). The relation between the five facets of mindfulness and worry in a non-clinical sample. Mindfulness, 3, 1521. https://doi.org/10.1007/s12671-011-0075-0.

Gu, J., Strauss, C., Bond, R., \& Cavanagh, K. (2015). How do mindfulness-based cognitive therapy and mindfulness-based stress reduction improve mental health and wellbeing? A systematic 
review and meta-analysis of mediation studies. Clinical Psychology Review, 37, 1-12. https://doi.org/10.1016/j.cpr.2015.01.006.

Heeren, A., Van Broeck, N., \& Philippot, P. (2009). The effects of mindfulness on executive processes and autobiographical memory specificity. Behaviour Research and Therapy, 47, 403-409. https://doi. org/10.1016/j.brat.2009.01.017.

Hodgins, H. S., \& Adair, K. C. (2010). Attentional processes and meditation. Consciousness and Cognition, 19, 872-878. https://doi.org/ 10.1016/j.concog.2010.04.002.

Holas, P., \& Jankowski, T. (2013). A cognitive perspective on mindfulness. International Journal of Psychology, 48, 232-243. https://doi. org/10.1080/00207594.2012.658056.

Hui, S. K. A., Wright, R. A., Stewart, C. C., Simmons, A., Eaton, B., \& Nolte, R. N. (2009). Performance, cardiovascular, and health behavior effects of an inhibitory strength training intervention. Motivation and Emotion, 33, 419-434. https://doi.org/ 10.1007/s11031-009-9146-0.

Jankowski, T., \& Holas, P. (2014). Metacognitive model of mindfulness. Consciousness and Cognition, 28, 64-80. https://doi.org/10.1016/j. concog.2014.06.005.

Jha, A. P., Krompinger, J., \& Baime, M. J. (2007). Mindfulness training modifies subsystems of attention. Cognitive, Affective, \& Behavioral Neuroscience, 7, 109-119. https://doi.org/10.3758/CABN.7.2.109.

Jha, A. P., Stanley, E., Kiyonaga, A., Wong, L., \& Gelfand, L. (2010). Examining the protective effects of mindfulness training on working memory capacity and affective experience. Emotion, 10, 54-64. https://doi.org/10.1037/a0018438.

Kabat-Zinn, J. (1990). Full catastrophe living: using the wisdom of your body and mind to face stress, pain and illness. New York: Delacorte Press.

Kabat-Zinn, J. (2003). Mindfulness-based interventions in context: past, present, and future. Clinical Psychology: Science and Practice, 10, 144-156. https://doi.org/10.1093/clipsy/bpg016.

Lau, M. A., Bishop, S. R., Buis, T., Anderson, N. D., Carlson, L., \& Carmody, J. (2006). The Toronto mindfulness scale: development and validation. Journal of Clinical Psychology, 62, 1445-1467. https://doi.org/10.1002/jclp.20326.

Lenth, R., Singmann, H., Love, J., Buerkner, P., \& Herve, M. (2018). Emmeans: estimated marginal means, aka least-squares means. The Comprehensive R Archive Network. https://cran.r-project.org/web/ packages/emmeans/index.html.

Lüdecke, D. (2018). sjPlot: data visuaslization for statistics in social science. Zenodo. https://doi.org/10.5281/zenodo.1308157.

Lykins, E. L. B., Baer, R., \& Gottlob, L. R. (2012). Performance-based tests of attention and memory in long-term mindfulness meditators and demographically matched nonmeditators. Cognitive Therapy and Research, 36, 103-114. https://doi.org/10.1007/s10608-010-9318-y.

Miyake, A., Friedman, N. P., Emerson, M. J., Witzki, A. H., Howerter, A., $\&$ Wager, T. D. (2000). The unity and diversity of executive functions and their contributions to complex "frontal lobe" tasks: a latent variable analysis. Cognitive Psychology, 41, 49-100. https://doi.org/ 10.1006/cogp.1999.0734.

Monsell, S. (2003). Task switching. Trends in Cognitive Sciences, 7, 134 140. https://doi.org/10.1016/S1364-6613(03)00028-7.

Polak, E. L. (2009). Impact of two sessions of mindfulness training on attention. (Doctoral dissertation). CiteSeerX. http://citeseerx.ist.psu. edu/viewdoc/download?doi=10.1.1.469.7226\&rep=rep1\&type=pdf

Prakash, R., Fountain-Zargoza, S., Kramer, A. F., Samimy, S., \& Wegman, J. (2018). Mindfulness and attention: current state-ofaffairs and future considerations. MindRxiv. https://doi.org/10. 31231/osf.io/8p3qv.

R Core Team (2014). R: a language and environment for statistical computing. R Foundation for Statistical Computing. http://www.Rproject.org/.

Raaijmakers, J. G. W., Schrijnemakers, J. M. C., \& Gremmen, F. (1999). How to deal with "the language-as-fixed-effect fallacy": common misconceptions and alternative solutions. Journal of Memory and Language, 41, 416-426. https://doi.org/10.1006/jmla.1999.2650.

Rogers, R. D., \& Monsell, S. (1995). Costs of a predictable switch between simple cognitive tasks. Journal of Experimental Psychology: General, 124, 207-231. https://doi.org/10.1037/ 0894-4105.20.6.675.

Salthouse, T. A. (1996). The processing-speed theory of adult age differences in cognition. Psychological Review, 103, 403-428. https://doi. org/10.1037/0033-295X.103.3.403.

Salthouse, T. A., Fristoe, N., McGuthry, K. E., \& Hambrick, D. Z. (1998). Relation of task switching to speed, age, and fluid intelligence. Psychology and Aging, 13, 445-461. https://doi.org/10.1037/08827974.13.3.445.

Schaefer, A., Nils, F., Sanchez, X., \& Philippot, P. (2010). Assessing the effectiveness of a large database of emotion-eliciting films: a new tool for emotion researchers. Cognition \& Emotion, 24, 1153-1172. https://doi.org/10.1080/02699930903274322.

Trezise, K., \& Reeve, R. A. (2016). Worry and working memory influence each other iteratively over time. Cognition and Emotion, 30, 353-368. https://doi.org/10.1080/02699931.2014.1002755.

Vandierendonck, A., Liefooghe, B., \& Verbruggen, F. (2010). Task switching: interplay of reconfiguration and interference control. Psychological Bulletin, 136, 601-626. https://doi.org/10.1037/ a0019791.

Watson, D., \& Clark, L. A. (1999). The PANAS-X: manual for the positive and negative affect schedule-expanded form. Iowa: University of Iowa.

Wenk-Sormaz, H. (2005). Meditation can reduce habitual responding. Advances in Mind-Body Medicine, 21, 33-49.

Publisher's Note Springer Nature remains neutral with regard to jurisdictional claims in published maps and institutional affiliations. 